\title{
The Transport In Medical Emergency - Simulation Study (TIME-Sim)
}

C. Clodi ${ }^{1}$, C. Schriefl ${ }^{1}$, M. Poppe ${ }^{1}$, A. M. Warenits,${ }^{1}$ A. Nuernberger ${ }^{1}$, M. Kießlinger ${ }^{2}$,

A. Zajicek², G. Klune ${ }^{2}$, M. Holzer ${ }^{1}$, C. Weiser ${ }^{1}$

1 Department of Emergency Medicine, Medical University of Vienna, Vienna General Hospital

2 Municipal Emergency Medical Service of the City of Vienna

\section{Background and Objective}

Extracorporeal cardiopulmonary resuscitation (eCPR) is a promising therapy for selected patients suffering from cardiac arrest (CA). A time period of 60 minutes from onset of CA until start of eCPR, appears to be a predictor of survival. In urban areas the most time-consuming and probably modifiable part is the Scene Time Interval (STI).

The purpose of this study was to find out, if a timeoptimized CPR-management is able to reduce the STI, without affecting the quality of CPR.

\section{Methods}

We carried out a prospective CA manikin simulation study including 4 different emergency medical service (EMS) teams (each staffed with one emergency physician). Each team passed 6 CA simulations as demonstrated in figure 1 .

In the control arm scenario of the CA simulation the EMS teams followed the standard ERC advanced life support (ALS) algorithm.

Then each team had a short introduction to a "time optimized CA scene strategy" - the intervention. It is basically based on immediate patient transfer onto a spineboard at the CA scene followed by ERC ALS standards and mechanically CPR usage as early as possible.

Further two CA simulation were passed by the teams applying the new strategy.

For further testing each team passed one control arm scenario as well as one intervention arm scenario one week later.

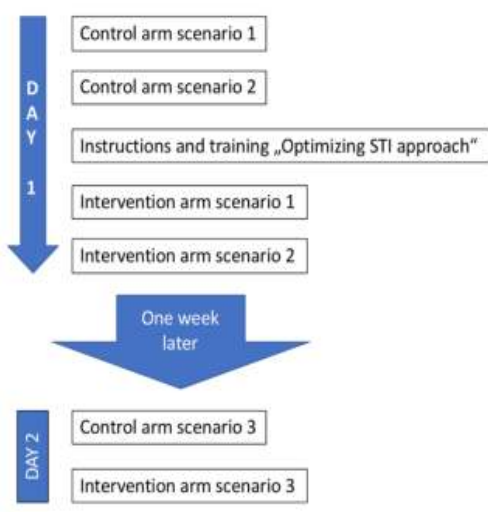

Figure 1: Sequence of Scenarios

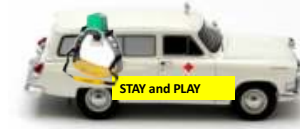

Figure 2: Load and go

\section{Results}

There was a significant reduction of the STI in the intervention arm (14:28 +/- 01:19 vs. 22:06 +/- 04:35, $\mathrm{p}=.001)$. Further there was a significantly earlier use of mechanical CPR $(1: 10+/-00: 27$ vs. $2: 56+/-1: 40, p=.004)$ and earlier start of transport to the ambulance car $(6: 10$ $+/-\quad 1: 27$ vs. $12: 44+/-3: 30, p=.001)$. Almost all parameters of quality of CPR did not differ between intervention and control scenarios (table 2).

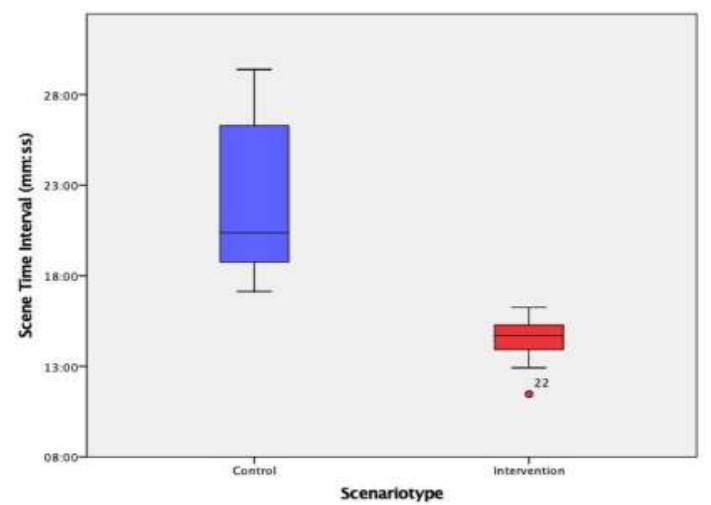

Figure 3: STI Control vs. Intervention Scenarios

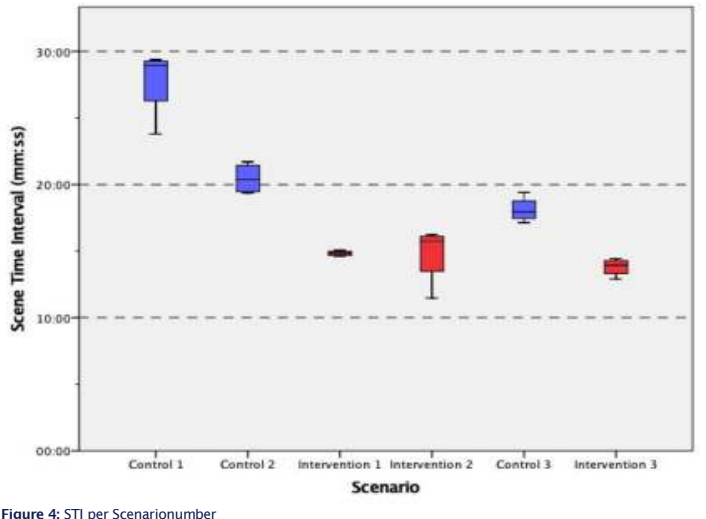

\begin{tabular}{|c|c|c|c|}
\hline & Control & Intervention & P-Value \\
\hline Time to mechanical CPR & $02: 56(01: 40)$ & $01: 10(00: 27)$ & .004 \\
\hline Time to decision to transport & $04: 30(02: 14)$ & $02: 13(00: 54)$ & .005 \\
\hline Time to rescue & $12: 44(03: 30)$ & $06: 10(01: 27)$ & .001 \\
\hline Table 1: Possible reasons for shorter STI & & & \\
\hline & Control & Intervention & P-Value \\
\hline Time to first shock (mm:ss) & $01: 24(00: 25)$ & $01: 27(00: 24)$ & .820 \\
\hline Compression fraction (\%) & $0,83(0,03)$ & $0,82(0,02)$ & .695 \\
\hline Mean no flow time (s) & $10(4)$ & $8(2)$ & .326 \\
\hline Mean compression depth (mm) & $54(9)$ & $51(8)$ & .481 \\
\hline Mean compression rate (bpm) & $107(2)$ & $109(1)$ & .055 \\
\hline Percentage of right position (\%) & $96(6)$ & $77(13)$ & .007 \\
\hline Mean tidal volume (ml) & $305(44)$ & $370(75)$ & .074 \\
\hline
\end{tabular}

\section{Conclusion}

By using a time-optimized strategy with focus on early transport it was possible to significantly reduce the STI enabling selected patients being treated with eCPR at a time point with higher odds for success. 